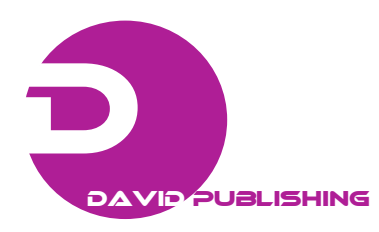

\title{
A Modified Methodology to Evaluate Viscosity of Non-Newtonian Liquid Foods with a Flow Channel Instrument
}

\author{
Masanori Yoshida ${ }^{1}$, Akimasa Soda ${ }^{2}$, Aya Togashi $^{2}$, Yuta Egawa ${ }^{2}$ and Yuki Sato ${ }^{2}$ \\ 1. Department of Applied Sciences, Muroran Institute of Technology, Muroran 050-8585, Japan \\ 2. Department of Chemistry and Chemical Engineering, Niigata University, Niigata 950-2181, Japan
}

Received: June 8, 2013 / Published: August 20, 2013.

\begin{abstract}
Through experimental analysis of the liquid flows in a flow channel instrument, prompt evaluation of the physically defined viscosity of non-Newtonian liquid foods was investigated for development into a structurally simple and easy-to-use viscometer. For Newtonian and non-Newtonian test liquids, a relation between the friction coefficient and Reynolds number, which was dimensionless as derived from an expression of analysis as a gravity current, indicated a condition under which the flow in the instrument was laminar and under which an average shear rate was on the order of less than $1 \mathrm{~s}^{-1}$. Prediction organized based on this empirical relation reproduced practically the flow curve determined for the liquid foods using a rotor type viscometer. Utilization of the channel instrument as a viscometer was formulated in terms of physical meanings of measurements such as the flow length and elapsed time.
\end{abstract}

Key words: Liquid food, non-Newtonian, viscosity, flow channel, flow analysis, viscometer.

\section{Introduction}

For control of liquid food quality, viscosity is an important evaluation item. Moreover, viscosity is a fundamental parameter that is necessary for design of unit operations in industrial production processes. Many liquid foods exhibit non-Newtonian behavior. Their viscosities change depending on the shear rate. Concretely, their viscosities differ along with variation in the speed of swallowing in the throat and the speed of transportation in process piping, which respectively engender differences of texture and power. To evaluate the viscosity of such non-Newtonian liquids definitively, we must analyze the relation between the shear stress and shear rate measured using a rotor type viscometer that is structurally complex and expensive. Additionally, practitioners in the fields of production and healthcare not specializing in fluid engineering,

\footnotetext{
Corresponding author: Masanori Yoshida, professor, research field: chemical engineering. E-mail: myoshida@mmm.muroran-it.ac.jp.
}

rheology, or other such areas might feel that analyses with repeated operations are cumbersome and consequently they require much time for viscosity evaluation. For prompt evaluation of the viscosity, design of an instrument and formulation of its usage, namely, construction of a system, is necessary for practitioners in relevant fields.

The search for easy operation has uncovered some unique instruments that are useful to evaluate the liquid food viscosity [1], all of which use gravity to flow a sample in the instrument and then evaluate its viscosity through measurement of the resultant flow length at the predetermined transit time. Such instruments commonly have a simple structure, which is a salient benefit. The flow length is usually regarded as a viscosity measure without complicated analysis. That is, the consistency is evaluated according to an empirical rule that the higher the liquid viscosity is, the shorter the flow length is. Such a principle used for viscosity evaluation lacks physical definition. The measure provides only a rough 
indication of the viscosity, which engenders the disadvantage of limited application for quality control and operational design. Resolving the cause of this disadvantage or defining the viscosity measure physically through theoretical or experimental analysis of the flow in the instrument might not merely expand the range of application of data; it might also provide a useful guideline for development of a structurally simple and easy-to-use instrument into a viscometer confirmed physically. For a Bostwick consistometer, which is an instrument that is widely used in food-related industries, particularly those producing tomato products, works have been undertaken to assess the physical implications of the measurements [2-12]. In these works, flows in the Bostwick consistometer have been analyzed fully from both theoretical and experimental viewpoints, and potentials of this instrument were demonstrated for prediction of the non-Newtonian rheological properties. A following step of work should be to make profitable use of the analytical accomplishments into prompt evaluation of the physically defined viscosity which is applicable not only to tomato products but also to many non-Newtonian liquid foods. To do so, it is necessary to reflect the analytical methodologies and to build a scheme for the viscosity evaluation, considering easy usage.

In this work, a flow channel instrument modeled after the Bostwick consistometer was made of transparent acrylic resin. Using this experimental apparatus, the flow in the instrument was monitored two-dimensionally to reflect the analytical methodologies available from reports of the literature. Concurrently, the time-courses of the flow length and liquid height were measured for sectional representatives of test liquids in travel. Then, a useful relation characterizing the flow in the instrument was sought. A scheme for the viscosity evaluation with that empirical relation was considered. Furthermore, reasonable application as a structurally simple and easy-to-use viscometer was discussed in comparison with a rotor type viscometer.

\section{Materials and Methods}

Glycerol and aqueous millet jelly solutions were used as Newtonian test liquids. Aqueous Xanthan gum solutions were adopted for use as non-Newtonian (shear thinning) test liquids. Tomato puree was used as a non-Newtonian model liquid food. The puree was heated with cornstarch and thickened by gelatinization of the starch. All liquids were handled at $25{ }^{\circ} \mathrm{C}$. Rheological properties of these liquids are listed in Table 1 . Density $\rho$, was measured using a pycnometer. Regarding viscosity, the change of the shear stress, $\tau$, was measured according to variation of the shear rate, $\gamma$, using a rotor type viscometer. For the Newtonian and non-Newtonian test liquids, which were regarded as homogeneous solutions, the cone-and-plate type was used. For the liquid foods, the parallel-plate type was used to reduce the impact of shear on the solids suspended in the liquid phase [1]. Thereby, parameters in the Herschel-Bulkley model (Eq. 1) were determined: the yield stress, $\tau_{0}$, the flow behavior index, $n$, and the consistency coefficient, $K$ :

$$
\tau / \dot{\gamma}=\eta=\tau_{0} \dot{\gamma}+\dot{K \gamma^{n-1}}
$$

Herein, $\eta$ is the viscosity for Newtonian liquids and the apparent viscosity for non-Newtonian liquids.

Fig. 1 portrays a flow channel instrument modeled after the Bostwick consistometer. The instrument as an experimental apparatus is made of transparent acrylic resin; the channel is $650 \mathrm{~mm}$ long, $40 \mathrm{~mm}$ high and 50 mm wide. Operation with the instrument was almost identical to that of the Bostwick consistometer. The samples were fully served at $25{ }^{\circ} \mathrm{C}$ into the holding compartment, which was surrounded by side walls, an end wall and a gate. The gate position was $50 \mathrm{~mm}$ in the flow direction from the end wall. The gate, being drawn upward, was released. Subsequently, the scene of the liquid flowing along the channel was observed using a video camera as viewed from the top and the back. The liquid flow was also checked with the side-viewing camera. From time-series images, measurements were made of the horizontal length between the tip of the flowing liquid and the end wall 
Table 1 Physical properties of liquids.

\begin{tabular}{llllll}
\hline Solute & Conc. $(\%)$ & $\rho\left(\mathrm{kg} / \mathrm{m}^{3}\right)$ & $K\left(\mathrm{~Pa} \mathrm{~s}^{n}\right)$ & $n(-)$ & $\tau_{0}(\mathrm{~Pa})$ \\
\hline Glycerol & 100 & 1,258 & 0.945 & 1 & - \\
Jelly & 85 & 1,358 & 1.29 & 1 & - \\
Jelly & 88 & 1,375 & 2.61 & 1 & - \\
Jelly & 91 & 1,394 & 7.51 & 1 & - \\
Jelly & 94 & 1,403 & 23.9 & 1 & - \\
\hline Xanthan & 0.5 & 999 & 1.68 & 0.323 & - \\
Xanthan & 0.7 & 1,000 & 3.13 & 0.251 & - \\
Xanthan & 0.9 & 1,001 & 4.80 & 0.200 & - \\
\hline 25\% tomato paste $+2.0 \%$ corn starch & 1,035 & 2.99 & 0.587 & 1.39 \\
25\% tomato paste $+3.0 \%$ corn starch & 1,035 & 3.30 & 0.554 & 2.96 \\
\hline
\end{tabular}
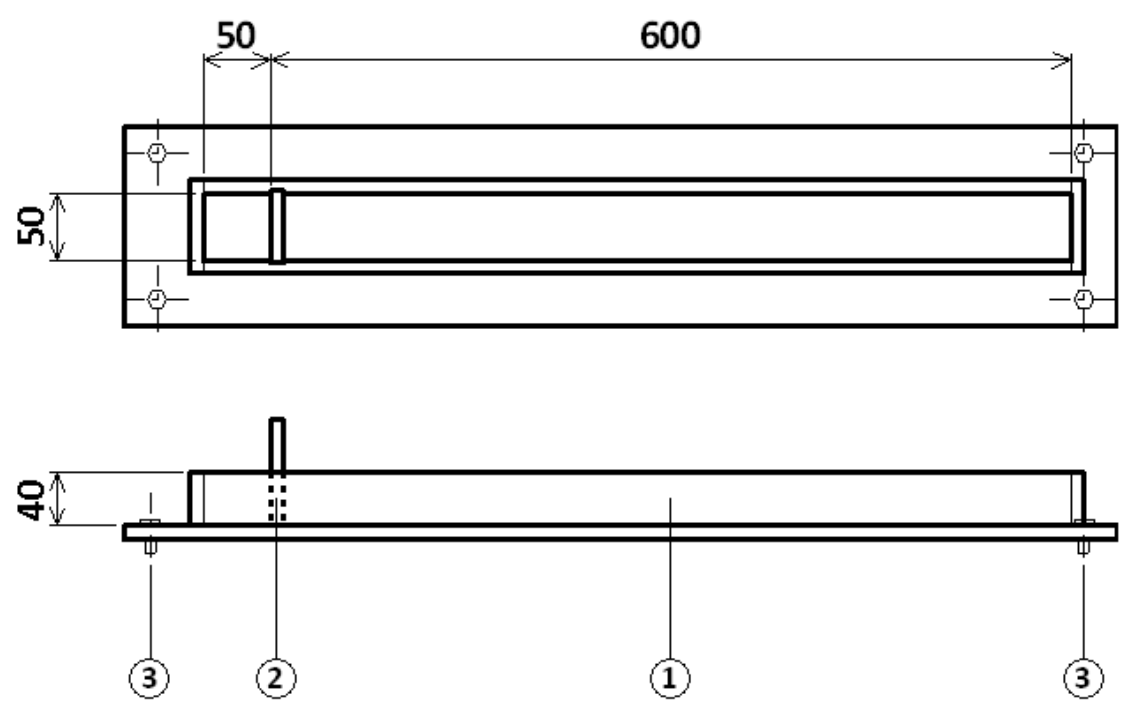

\section{1. flow channel, 2. gate, 3. leveler}

Fig. 1 Schematic diagram of flow channel instrument used as experimental apparatus (dimensions in millimeters).

corresponding to the back wall and the liquid heights at various horizontal positions including the back wall position.

\section{Results and Discussion}

\subsection{Flows of Test Liquids in the Instrument}

According to a survey by Perona [13], for the problem of possible correlation of the viscosity measure by the Bostwick consistometer, namely, the Bostwick consistency, with the viscosity or apparent viscosity, conclusions among researchers have shown agreement and discrepancies after the first debates by Eolkin [14], who commented by an undoubtedly different direction moved McKarthy and Seymour [6, 7]. The flow in the instrument was simplified to a one-dimensional gravity current, which can be described theoretically by a solution. Piau and Debiane [9] and Balmforth et al. [12] introduced the idea of gravity current as a part of their analytical works. The accomplishment obtained from such an analysis including experimental verifications allowed correlation of the Bostwick consistency with the viscosity or apparent viscosity. Theoretical bases with respect to the gravity current in a flow channel were given by Didden and Maxworthy [15], Huppert [16], Di Federico et al. [17] and others. First, the difference of flow behavior as ascertained through experimentation and theory should be examined closely.

In theoretical analysis, it was assumed that a liquid is homogeneous and that the effect of surface tension of 
the liquid and the momentum transfer on the surface are negligible. Additional assumptions were made that the liquid adheres perfectly to the bottom and back walls of the instrument and that the side walls play no role. The gravity current analysis expresses the flow length, $L$, corresponding to the Bostwick measurement plus the length of the holding compartment, as a function of time [17].

$L=\xi_{\mathrm{N}}[n /(2 n+1)]^{n /(2 n+3)}(\rho g / K)^{1 /(2 n+3)} q^{(n+2) /(2 n+3)} t^{n /(2 n+3)}(2)$ herein, $g$ is the acceleration of gravity, $q$ is the liquid volume per unit width, $\rho$ is the liquid density, $n$ is the flow behavior index, $K$ is the consistency coefficient, $t$ is the time and $\xi_{\mathrm{N}}$ is given as a function of $n$. In this analysis, the liquid heights at various horizontal positions are also obtained as a function of time [17]. Characteristically, the height at the position of the back wall of the instrument, $h_{0}$, is expressed as below:

$$
h_{0}=\xi_{\mathrm{N}}{ }^{(n+1) /(n+2)}[(2 n+1) / n]^{n /(2 n+3)}[n /(2 n+3)]^{n /(2 n+1)}
$$

$\times[(n+2) /(n+1)]^{1 /(2 n+1)}(\rho g / K)^{-1 /(2 n+3)} q^{(n+1) /(2 n+3)} t^{-n /(2 n+3)}(3)$

Based on Eqs. 2 and 3, analyses of the flows in the instrument were first made for Newtonian and non-Newtonian test liquids. Fig. 2a shows analytical results of $L$ and those of $h_{0}$ as a function of $t$ for the Newtonian liquids. The lines in the figure respectively represent the $L$ values predicted from Eq. 2 and the $h_{0}$ values predicted from Eq. 3 . The tendency for $L$ to increase and that for $h_{0}$ to decrease over time were illustrated to be described successfully in terms of the power of time in Eqs. 2 and 3. Their values at any time differed between measurement and prediction. That is, in the prediction, the $L$ values were overestimated and $h_{0}$ values were underestimated. Such an offset seems to result from the flow immediately after release of the gate. In the immediate time period, the flow is dominated by the inertial force rather than the viscous force. Additionally, the gate opened upward locally generates the flow which is opposite to the gravity, of which the magnitude differs easily depending on the operation of practitioners [8]. These phenomena render the gravity current analysis as approximate because the difference in the early period affects the following coincidence. Fig. 2b shows the results for the non-Newtonian liquids. For $L$ and $h_{0}$, respectively, differences between the experimental and predicted values were tremendous in the immediate time period and are apparently followed by courses affected by the early differences. The remarkable differences in the non-Newtonian liquids are addressed considering possible elasticity of the liquids. The non-Newtonian test liquids used in this work have the elasticity on one level or another as well as the viscosity. Release of the gate allows the liquid to deform rapidly and liquid under such a condition would dominantly exhibit elasticity relative to the viscosity [18]. A reduced viscous resistance to the flow [19] can affect the considerable increase of $L$ and decrease of $h_{0}$.

For the phenomenon immediately after release of the gate, results obtained not through the gravity current analysis but through slump analysis have been presented to facilitate the utilization of the instrument as a viscometer [9, 12]. Theoretical bases with respect to slumping of the gravity current in a flow channel were given by Huppert and Sinpson [20], and others. Such results, being informative, are also expressed as a function of time. The slump analysis might be helpful for evaluation of the other rheological properties such as the yield stress rather than the viscosity [10]. Here, emphasizing the fact that the operation releasing the gate is practically unavoidable, an analytical methodology for which the expression has no time term was regarded as treating the data with the effect of phenomena in the early period subtracted.

As presented in Fig. 2, the increase of $L$ and decrease of $h_{0}$ proceed in mutual relation. Combining Eqs. 2 and 3 yields the following equation without the time term. $L h_{0}=\xi_{\mathrm{N}}{ }^{(2 n+3) /(n+2)}\{n /(2 n+3)\}^{n /(2 n+1)}\{(n+2) /(n+1)\}^{1 /(2 n+1)} q$

Eq. 4 expresses the relation between $h_{0}$ and $L$ as determined by the flow behavior index $n$, and the liquid volume in instrument, $q$. Fig. 3 shows plots of $h_{0}$ against $L$ for the Newtonian and non-Newtonian test liquids. The index, $n$, for the test liquids ranged from 


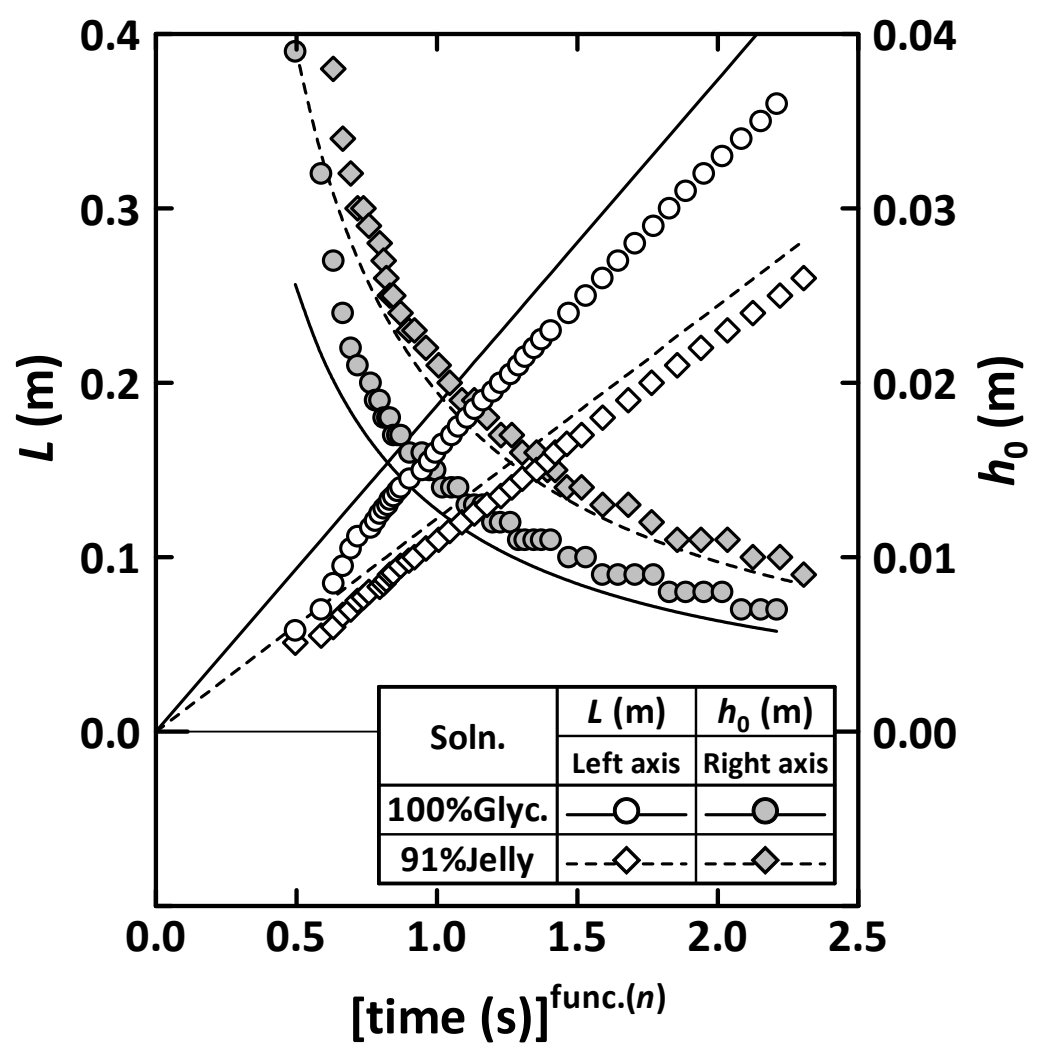

Fig. 2a Time-courses of flow length, $L$, and those of backend height, $h_{0}$, for Newtonian test liquids.

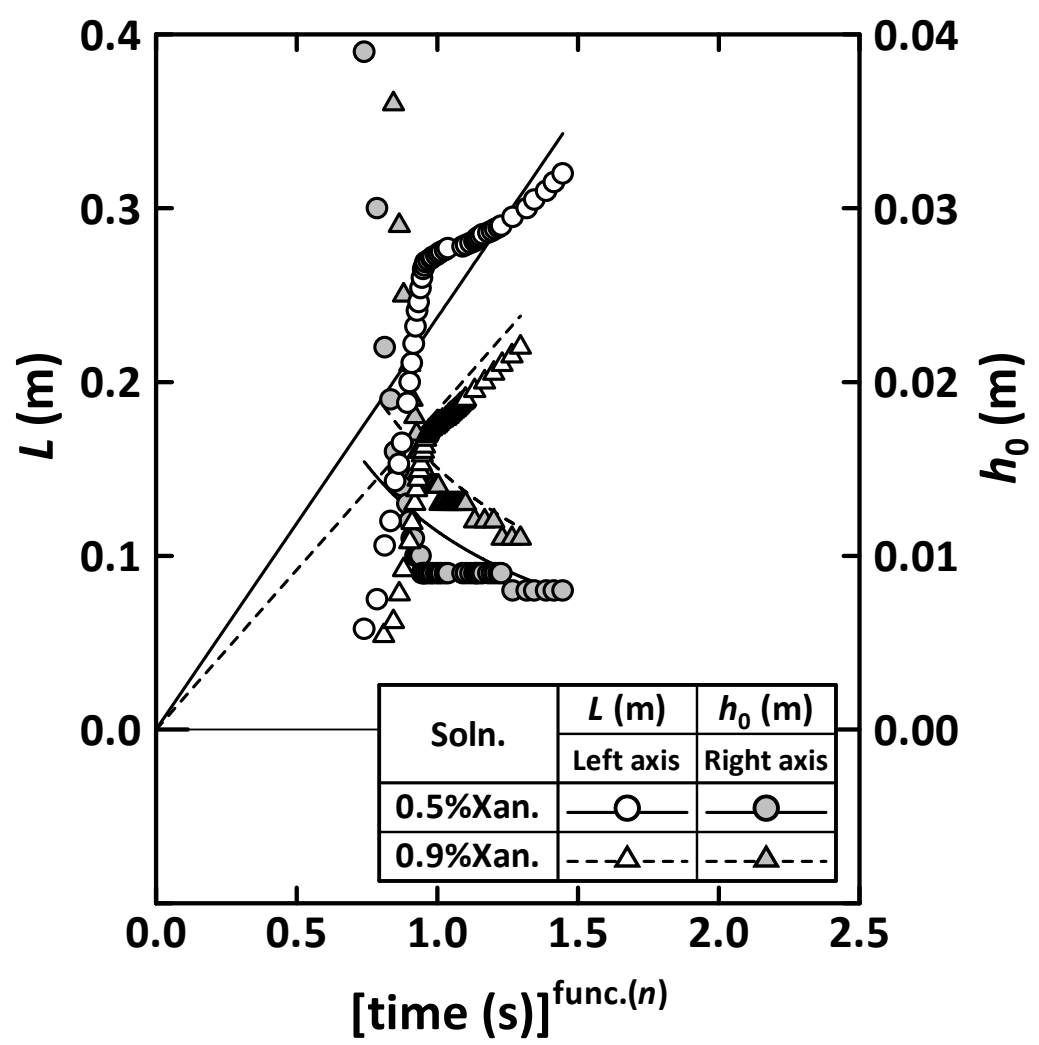

Fig. 2b Time-courses of flow length, $L$, and those of backend height, $h_{0}$, for non-Newtonian test liquids. 
0.20 to 1.00 . For the minimum and maximum values of $n$, respectively, predictions were made with Eq. 4 . The results are represented by the dashed and solid lines in the figure. According to the predictions with Eq. 4, the relation between $h_{0}$ and $L$ differs depending on $n$. In the experiments, such a difference could not be observed with an unsatisfactory coincidence between the measured values and those calculated particularly for the non-Newtonian test liquids. This is apparently a reflection of approximate gravity current analysis in the time period immediately after release of the gate, as described above. However, Fig. 3 presents an illustration of an empirical relation between $h_{0}$ and $L$ that is practically applicable for Newtonian and non-Newtonian liquids, irrespective of $n$. In this work, the following relation was assumed at the sacrifice of a less theoretical basis:

$$
h_{0} L=C_{\mathrm{q}}
$$

Coefficient $C_{\mathrm{q}}$ which depends only on $q$ was determined to be $0.0024 \mathrm{~m}^{2}$. For analysis of the liquid flow in this instrument, both the flow length and the backend height are physical quantities that have important implications. The latter is not easier to measure experimentally because the change of $h_{0}$ is smaller than that of $L$ with progress of the flow phenomena. Eq. 5 can be used to predict $h_{0}$ values with $L$ values measured experimentally.

In addition to the flow length and the backend height, a flow rate is required for evaluation of the liquid flow in this instrument. The change of flow length with time, $d L / d t$, was selected as a flow rate. Fig. 4 shows plots of $d L / d t$ against the flow length, $L$. With progress of the flow phenomena, $d L / d t$ tended to decrease, with the exception of the range for $L$ to have smaller values corresponding to the time period immediately after release of the gate. The decrease of $d L / d t$ for the Newtonian test liquids was straightforward. For the non-Newtonian test liquids, its decrease was stepwise and a distinct range was observed, although the values were larger and the change was smaller. Existence of such a range appears to support solid-like behavior of the non-Newtonian liquids used in this work, as suggested in consideration for Fig. 2b. In the following $L$ range, the tendencies to decrease exponentially were similar to those for the Newtonian liquids. For practical utilization of the instrument, the period after sometime elapsed, namely, the range for $L$ to have larger values, is preferred because of the ease of measuring the length with flow progressing. Consequently, analysis was forwarded for the respective $L$ ranges where a decelerating tendency was observed for both the Newtonian and non-Newtonian liquids, as indicated by dotted lines in Fig. 4.

\subsection{Characterization of Liquid Flows in the Instrument}

In keeping with results in the previous section, characterization of the flow of liquid in the instrument was then implemented in a hydraulic manner that is both conventional and comprehensive. Fig. 5 portrays a section of the liquid flowing along the channel in the $x-z$ coordinate system. The following dimensionless parameters were defined [21]:

$$
\begin{aligned}
& \text { Friction coefficient: } C_{\mathrm{f}}=\tau /\left(\rho U_{\mathrm{c}}{ }^{2} / 2\right) \\
& \text { Reynolds number: } R e=\rho U_{\mathrm{c}} D_{\mathrm{c}} / \eta
\end{aligned}
$$

where, $\tau$ is the shear stress, $\rho$ is the liquid density, and $\eta$ is the liquid viscosity. Two variables, $U_{\mathrm{c}}$ and $D_{\mathrm{c}}$, are a characteristic velocity and a characteristic length, respectively. Essentially, the average velocity should be employed for $U_{\mathrm{c}}[22,23]$. In consideration of the ease of determination, the time change of flow length, $d L / d t$, was utilized. Additionally, the hydraulic diameter should be adopted for $D_{\mathrm{c}}[22,23]$. Because that diameter changes in a direction of the flow, backend height, $h_{0}$, involved in the diameter, was allowed to be a representative of $D_{\mathrm{c}}$. From the horizontal force balance, the average shear stress, $\tau_{\mathrm{av}}$, is expressed with flow length, $L$, and acceleration of gravity, $g$, as below:

$$
\tau_{\mathrm{av}}=\rho g h_{0}^{2} / 2 L
$$

Representing $\tau$ in Eq. 6 by $\tau_{\text {av }}$ and applying the characteristic parameters to Eqs. 6 and 7 yield the respective equations, rewritten as the following: 

Foods with a Flow Channel Instrument

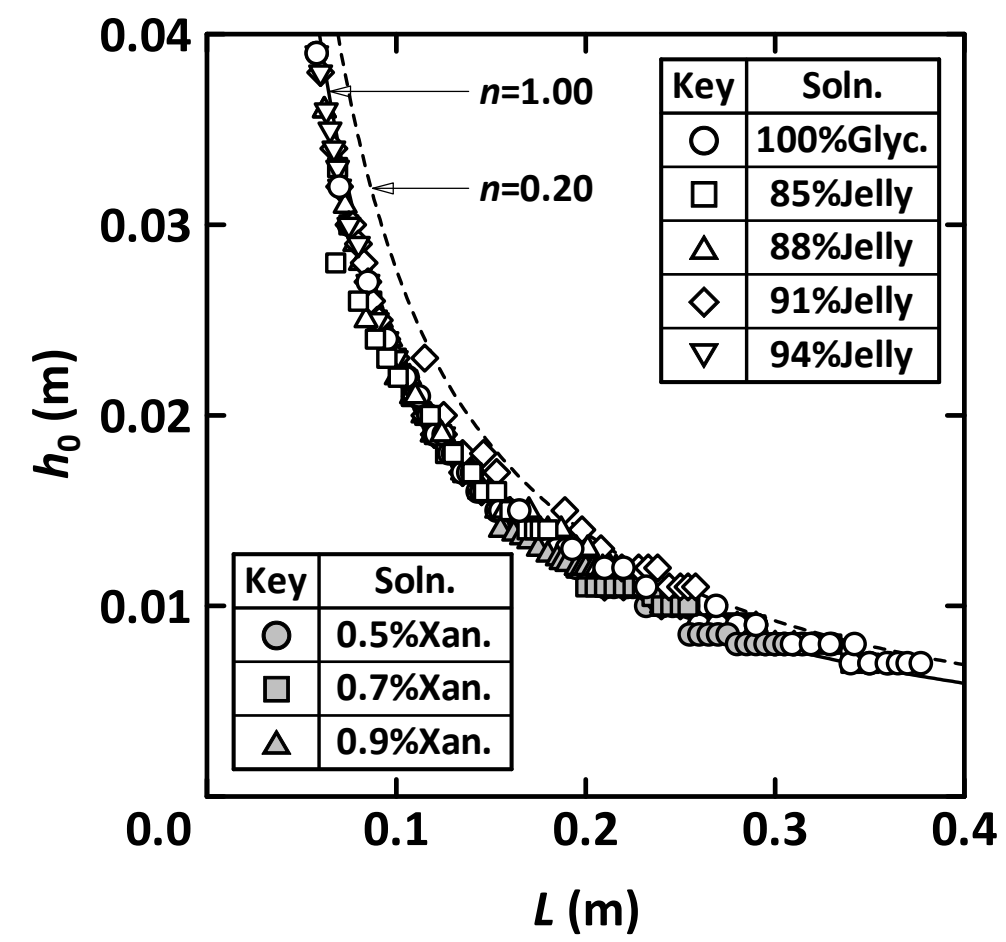

Fig. 3 Change of backend height, $h_{0}$, as viewed from change of flow length, $L$.

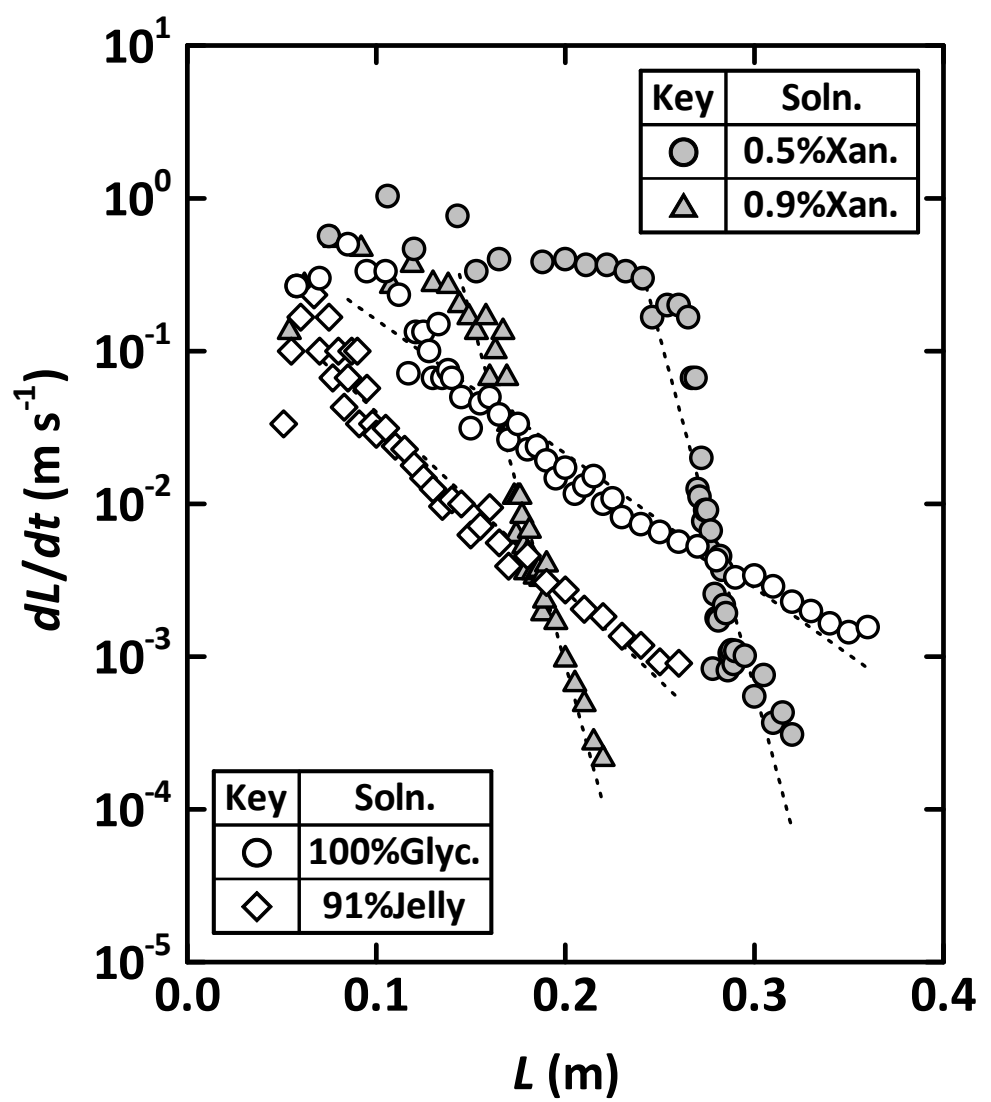

Fig. 4 Change of flow rate, $d L / d t$, as viewed from change of flow length, $L$. 


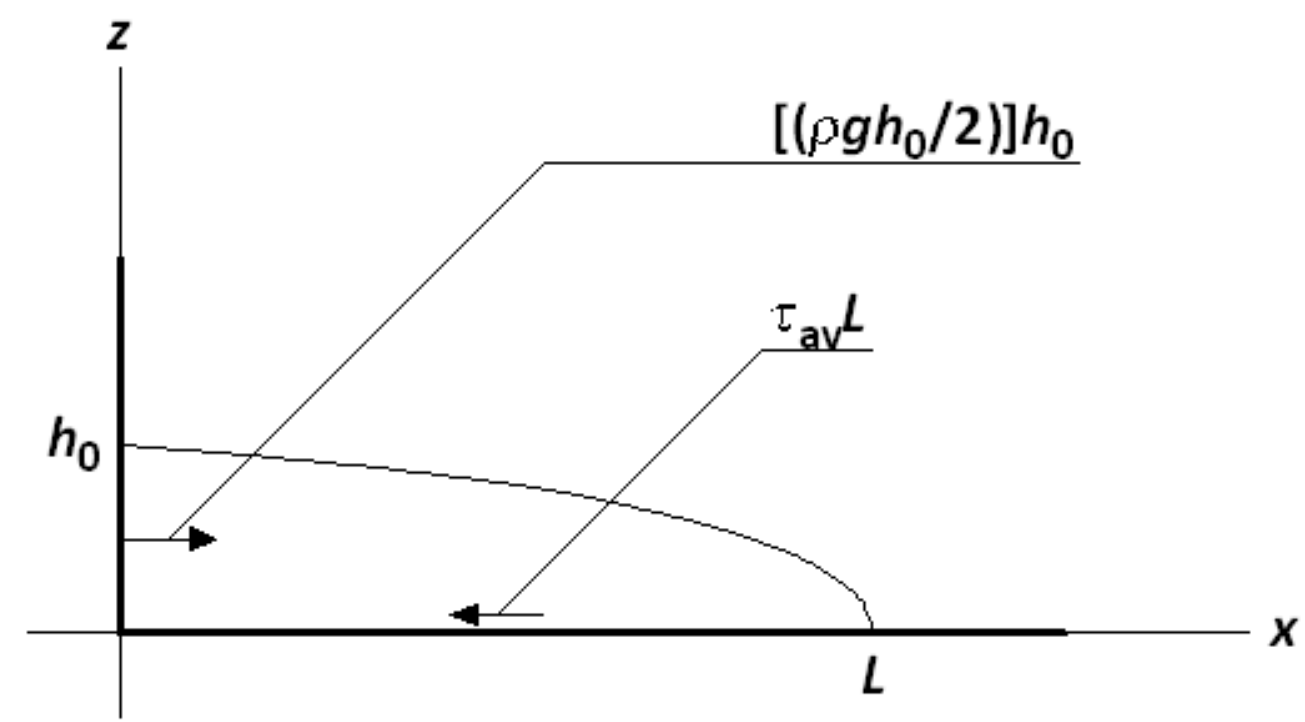

Fig. 5 Sectional view of viscous liquid in flow channel instrument and definition of $x$-z coordinate system.

$$
\begin{aligned}
& C_{\mathrm{f}}=\tau_{\mathrm{av}} /\left[\rho(d L / d t)^{2} / 2\right] \\
& R e=\rho(d L / d t) h_{0} / \eta
\end{aligned}
$$

Fig. 6 shows analytical results as a relation between $C_{\mathrm{f}}$ and $R e$ for the Newtonian test liquids. In fact, $C_{\mathrm{f}}$ was inversely proportional to $R e$, which indicated that the flow in the instrument was laminar flow dominated by the viscous force. Thereby, $C_{\mathrm{f}}$ can be related to $R e$ as:

$$
C_{\mathrm{f}}=7.0 \operatorname{Re}^{-1}
$$

Eq. 9 is expected to be the relation that is applicable also for similar instruments with the requirement of $R e$ $<10$, while further experiments with variation of the geometrical conditions of instrument are necessary for validation of the equation obtained with an experimental apparatus in this work. The laminar flow constant in the $C_{\mathrm{f}}$ vs. $R e$ relationship was 7.0 in this analysis as mentioned above. Burger et al. [23] presented the values for the laminar flow in open channels which were comparable to that in closed circular pipes as 16 . Because the respective definitions of $C_{\mathrm{f}}$ and $R e$ differ between Burger et al. and this works, rigorous comparison is impossible. In rough comparison, when the hydraulic diameter is assumed to be $2 h_{0}$ for the flow system in this work, the constant of 7.0 is transformed into 14. Additionally, employment of the average velocity instead of $d L / d t$ for $U_{\mathrm{c}}$ would bring the value of 14 close to 16 with undervalued $U_{\mathrm{c}}$.

For non-Newtonian test liquids, the viscosity changes with the flow process depending on the shear rate, $\dot{\gamma}$. Therefore, evaluation of $\dot{\gamma}$ in the instrument is necessary in analysis as described above. A characteristic shear rate, $\dot{\gamma}_{\mathrm{c}}$, was expressed with the characteristic velocity and length, $U_{\mathrm{c}}$ and $D_{\mathrm{c}}$, as follows [24]:

$$
\dot{\gamma}_{\mathrm{c}}=C_{\mathrm{c}}\left(U_{\mathrm{c}} / D_{\mathrm{c}}\right)=C_{\mathrm{c}}\left[(d L / d t) / h_{0}\right]
$$

The coefficient $C_{\mathrm{c}}$ was determined with the experimental data for the Newtonian test liquids. That is, combining Eqs. 8 and 10 with Eq. 1, as:

$$
\eta=\tau_{\mathrm{av}} / \dot{\gamma}_{\mathrm{c}}=\left(1 / C_{\mathrm{c}}\right)(1 / 2)(\rho g)\left[h_{0}{ }^{3} / L(d L / d t)\right]
$$

The term shown in the right-hand bracket of Eq. 11 is calculable using the experimental measurements in the instrument. When the measurement with the cone-and-plate viscometer is used for $\eta$ in the left-hand side, $C_{\mathrm{c}}$ can be determined for the shear rate based on Eq. 10. For $C_{\mathrm{c}}$ being practically unchanged depending on the viscosity and the flow process, its value was assessed as 3.5. Thereby, Eq. 10 was rewritten as:

$$
\dot{\gamma}_{c}=3.5\left[(d L / d t) / h_{0}\right]
$$

The term in the right-hand bracket of Eq. 10' is an average shear rate evaluated between the upper free surface and the lower rigid surface of the liquid flowing along the channel. Eq. 10' expresses that the shear rate characterizing the flow in the instrument is three and a half times larger than the average rate. The velocity gradient is regarded as larger in a region near the 


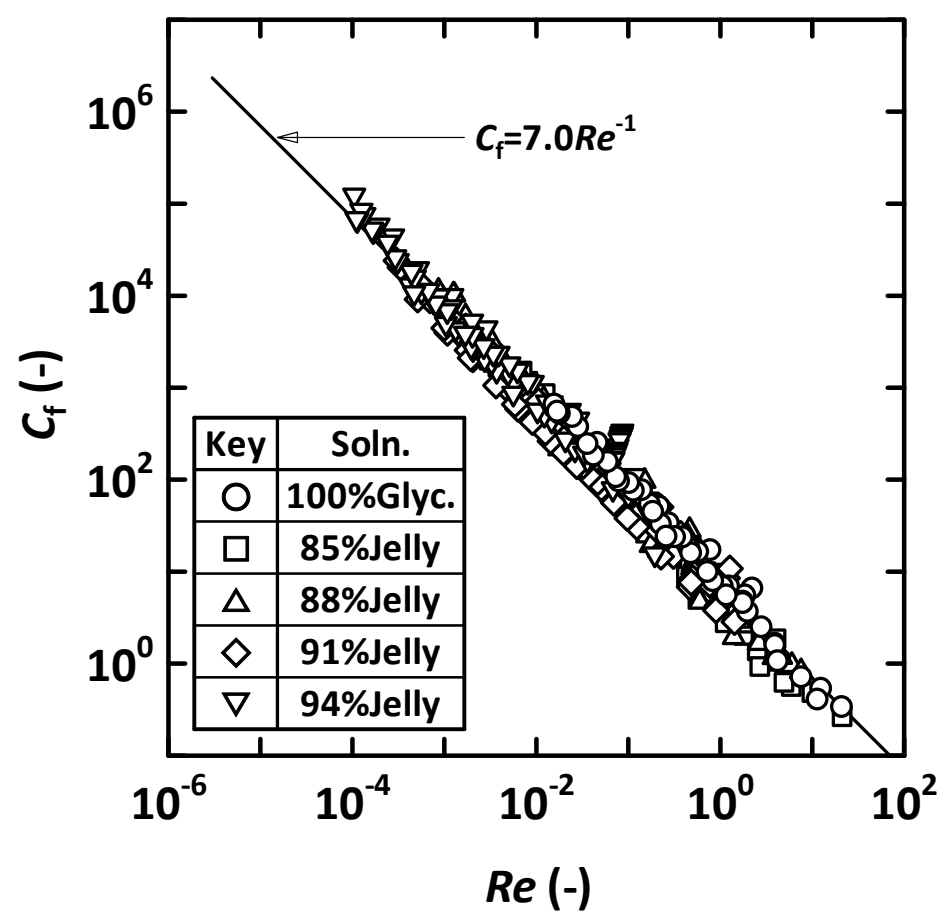

Fig. 6 Relation between friction coefficient, $C_{\mathrm{f}}$, and Reynolds number, $R e$, for Newtonian test liquids.

bottom wall of the instrument. Therefore, $\dot{\gamma}_{c}$ over the average rate can be explained because the flow in such a region dominantly influences the overall flow. In this work, applying $\gamma_{c}$ obtained by Eq. 10 ' to Eq. 1, the apparent viscosity, $\eta$, for the non-Newtonian test liquids was calculated.

Fig. 7 shows a relation between $C_{\mathrm{f}}$ and $R e$ for the non-Newtonian test liquids. The solid line in the figure represents the values calculated from Eq. 9 for the Newtonian test liquids. The relation analyzed as described above for the non-Newtonian test liquids was regarded as almost identical to that for the Newtonian test liquids. In the $R e$ range of about $1-10$, the similarity was rather insufficient. The applicable range of $R e$ in Eq. 9 should be regarded as one or less. Under such a condition, $\gamma_{c} s$ calculated from Eq. 10' were under about $1 \mathrm{~s}^{-1}$. This instrument is expected to be useful for measurement of the apparent viscosity in such a shear rate condition.

\subsection{Development of the Instrument into a Viscometer and Viscosity Evaluation for Liquid Foods}

Using the empirical relation of Eq. 9, building of a scheme was attempted for evaluation of the apparent viscosity in conjunction with the shear rate. A diagram of the scheme is presented in Fig. 8. For a sample with known density, flow length, $L s$, is measured at the appropriately defined elapsed times. The flow rate is calculated as the differential of $L$ with respect to the time, $d L / d t$ and the backend height, $h_{0}$, is calculated from Eq. 5. Applying $d L / d t$ and $h_{0}$ to Eqs. 6' and 8, the friction coefficient $C_{\mathrm{f}}$ is determined. Then the Reynolds number, $R e$, is evaluated using Eq. 9. Data meeting the conditions of $R e<1$ are treated. Whereas, the apparent viscosity, $\eta$, is obtained with the definition of $R e$, Eq. 7', the characteristic shear rate, $\dot{\gamma}_{\mathrm{c}}$, is obtained with Eq. 10'.

For suspensions of tomato paste and corn starch, measurements were then made of the flow lengths at different elapsed times. The flow channel instrument has some problems affecting its measurements. Solid-liquid separation in suspension, which is one problem, was insignificantly observed for the suspensions used in this work. Although this problem should be considered rigorously [11], we avoided describing many details because of a priority on demonstration of the scheme 


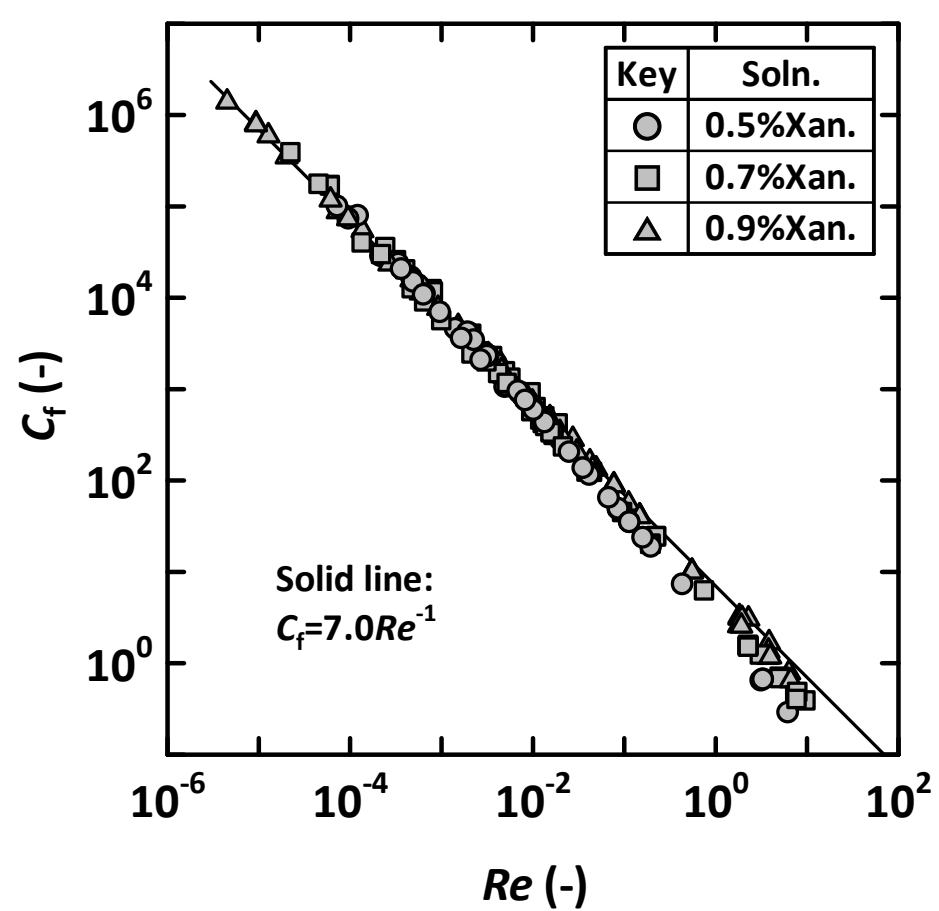

Fig. 7 Relation between friction coefficient, $C_{\mathrm{f}}$, and Reynolds number, $R e$, for non-Newtonian test liquids.

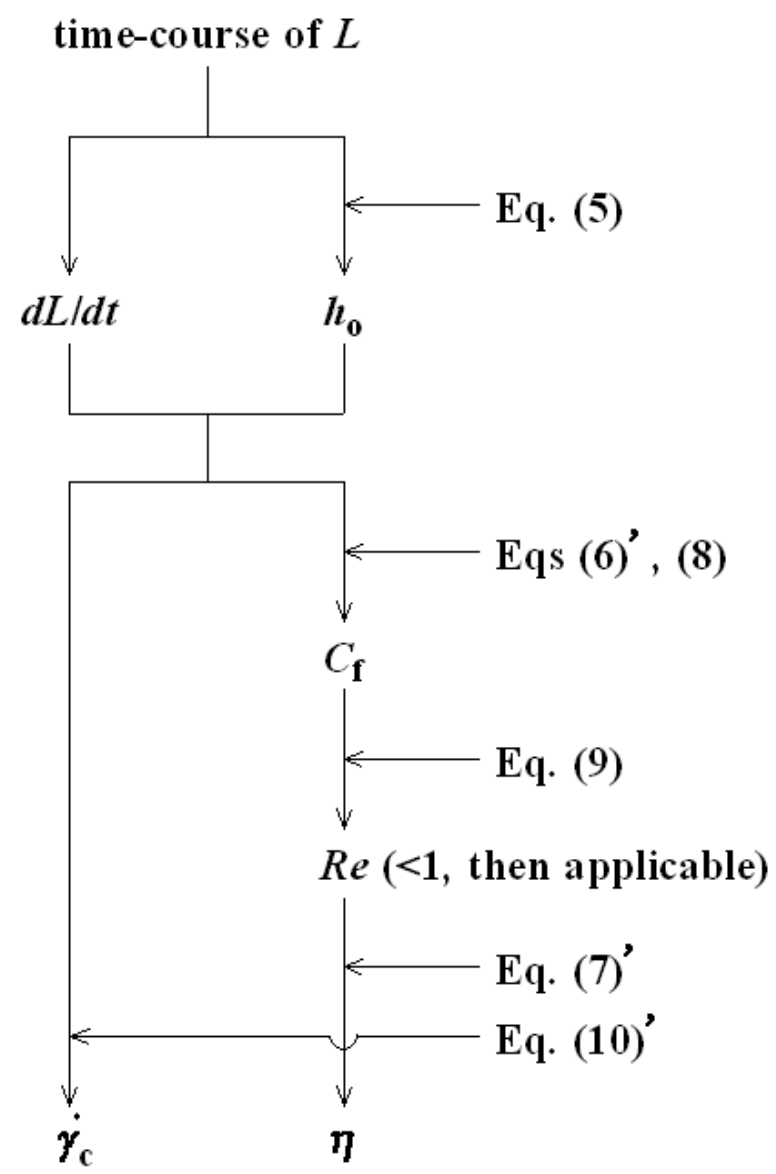

Fig. 8 Flow diagram of scheme for evaluation of viscosity with flow channel instrument. 
presented in Fig. 8. Plots of the apparent viscosity evaluated through the above scheme against the characteristic shear rate are shown in Fig. 9. Concurrently, through measurement with the parallel-plate viscometer, changes of the apparent viscosity of sample were evaluated along with variation of the shear rate. In Fig. 9, the latter relations are represented as solid and dashed lines. Accordance between the measurements with the flow channel instrument and those with the rotor type viscometer was acceptable for practical utilization of that instrument as a viscometer. These results demonstrated the possibility that the flow channel instrument evaluates the physically defined viscosity with adequate accuracy, leading to development of a structurally simple viscometer.

As shown in Fig. 9, for the liquid foods used in this work, the shear rate was determined to range from 0.1 $\mathrm{s}^{-1}$ to $1 \mathrm{~s}^{-1}$. Such a range is covered in examination with the test liquids. Therefore, application of the scheme is judged to be valid. However, this exemplification means that the flow channel instrument is limited to a lower range of shear rates when leveling or pouring liquid foods are of concern. In other words, the flow channel instrument might not evaluate the viscosity appropriately under a higher condition of shear rate for liquid foods when one is eating and drinking them, or industrially processing them. Although the shear rate formed in the instrument is dependent on the liquid food thickness, the mechanism of liquid flow attributable to the instrument structure is regarded as determinative for the limited range of shear rate. It must be pointed out that a structurally simple instrument should be developed to evaluate the viscosity over widely diverse shear rates.

The scheme described above enables the structurally simple flow channel instrument to determine the flow curve of a sample for a limited range of shear rates. However, in point of relative comparison of the viscosity among samples, ease of use that increases convenience and reduces trouble is preferable. In some situations, promptness, which saves time, is required. Quality control of liquid foods that is pursued using structurally simple instruments such as a Bostwick consistometer has been conducted conventionally with the flow length at the predetermined transit time. As

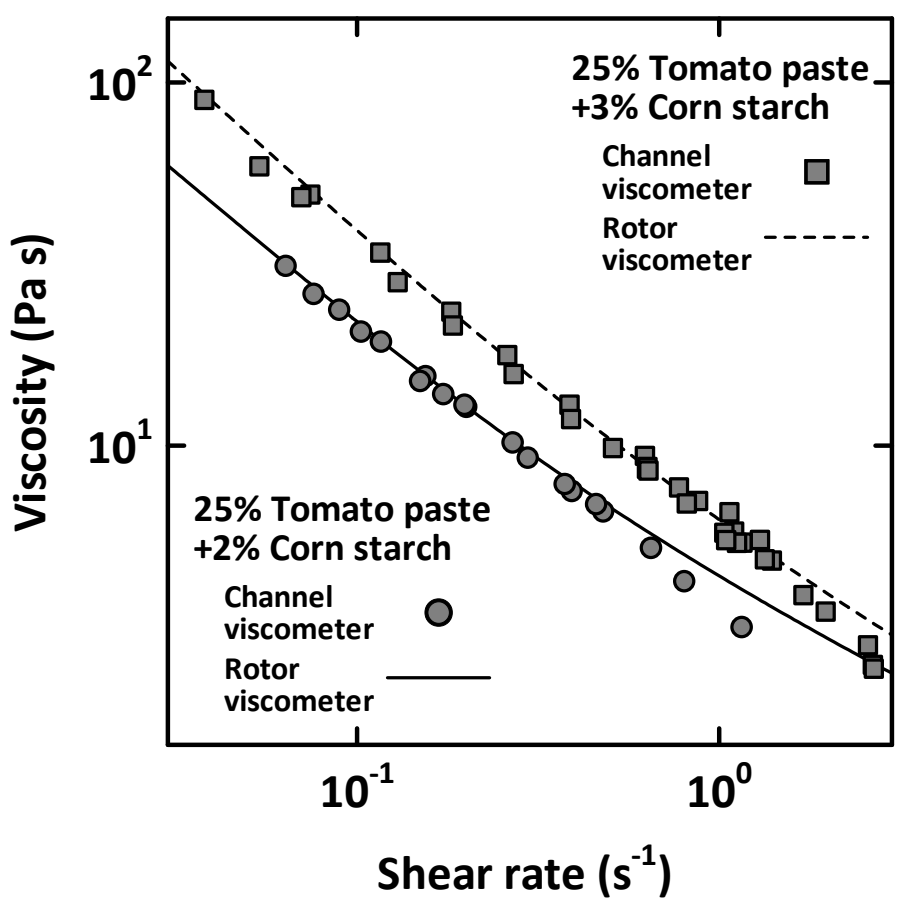

Fig. 9 Comparison of viscosity to the shear rate between the channel and rotor viscometers. 
expressed by Eq. 10', the shear rate, $\gamma_{c}$, is a function of the flow length and rate, which elucidates an aspect that the viscosity is compared under different shear rate conditions in the conventional rule. In contrast, comparison of the elapsed time at the specific flow length is made under the common conditions of shear stress, where $\dot{\gamma}_{\mathrm{c}}$ differs, because the stress, $\tau_{\mathrm{av}}$, expressed by Eq. 8 is dependent on the length alone. Identical shear stress generated in the liquid flowing along the channel is regarded as implying a similar shape of the liquid section. Consequently, evaluation of the viscosity in the scheme described above can be reduced to measurement of the elapsed time at the specific flow length, remaining the most important meaning. In addition to the foregoing scheme, its reduction for possibly prompt evaluation of the viscosity is proposed as a direction for easy use of the flow channel instrument.

\section{Conclusions}

To develop a flow channel instrument into a structurally simple and easy-to-use viscometer, flows of Newtonian and non-Newtonian test liquids in the instrument were analyzed experimentally. The following findings were obtained with respect to evaluation of the viscosity for non-Newtonian liquid foods.

The flow in the instrument was characterized as a relation between the friction coefficient and the Reynolds number in a dimensionless form to which an expression of analysis as gravity current was modified.

The test liquids used in this work behaved in a laminar condition where the flow rate decreased exponentially for both Newtonian and non-Newtonian liquids, characteristic shear rates being on the order of less than $1 \mathrm{~s}^{-1}$.

Based on the empirical relation between the friction coefficient and the Reynolds number, a scheme to evaluate the physically defined viscosity was presented for liquid foods.

For prompt evaluation of the viscosity, measurement of the elapsed time at a specific flow length is recommended.

\section{Acknowledgments}

The authors are grateful for financial support from a Grant-in-Aid for Scientific Research (C) from the Ministry of Education, Culture, Sports, Science and Technology of Japan.

\section{References}

[1] T.G. Mezger, The Rheology Handbook, Vincentz Network, Hannover, Germany, 2006, pp. 188-189, 199-206.

[2] M.A. Rao, M.C. Bourne, Analysis of the plastometer and correlation of Bostwick consistometer data, J. Food Sci. 42 (1977) 261-264.

[3] G.L. Marsh, J.E. Buhlert, S.J. Leonard, Effect of composition upon Bostwick consistency of tomato concentrate, J. Food Sci. 45 (1980) 703-706.

[4] N. Takada, P.E. Nelson, A new consistency method for tomato products: The precipitate weight ratio, J. Food Sci. 48 (1983) 1460-1462.

[5] M.C.M. Vercruysse, J.F. Steffe, Online viscometry for pureed baby food: Correlation of Bostwick consistometer readings and apparent viscosity data, J. Food Process Eng. 11 (1989) 193-202.

[6] K.L. McCarthy, J.D. Seymour, A fundamental approach for the relationship between the Bostwick measurement and Newtonian fluid viscosity, J. Texture Studies 24 (1993) 1-10.

[7] K.L. McCarthy, J.D. Seymour, Gravity current analysis of the Bostwick consistometer for power law foods, J. Texture Studies 25 (1994) 207-220.

[8] S.A. Barringer, A.T.M.S. Azam, B. Heskitt, S. Sastry, Online prediction of Bostwick consistency from pressure differential in pipe flow for ketchup and related tomato products, J. Food Processing Preservation 22 (1998) 211-220.

[9] J.M. Piau, K. Debiane, Consistometers rheometry of power-law viscous fluids, J. Non-Newtonian Fluid Mech. 127 (2005) 213-224.

[10] R.R. Milczarek, K.L. McCarthy, Relationship between the Bostwick measurement and fluid properties, J. Texture Studies 37 (2006) 640-654.

[11] M.M. Tehrani, A. Ghandi, Modification of Bostwick method to determine tomato concentration consistency, J. Food Eng. 79 (2007) 1483-1486.

[12] N.J. Balmforth, R.V. Craster, P. Perona, A.C. Rust, R. Sassi, Viscoplastic dam breaks and the Bostwick consistometer, J. Non-Newtonian Fluid Mech. 142 (2007) 
Foods with a Flow Channel Instrument

63-78.

[13] P. Perona, Bostwick degree and rheological properties: An up-to-date viewpoint, Appl. Rheol. 15 (2005) 218-229.

[14] D. Eolkin, The plastometer: A new development in continuous recording and controlling consistometer, Food Technol. 11 (1957) 253-257.

[15] N. Didden, T. Maxworthy, The viscous spreading of plane and axisymmetric gravity currents, J. Fluid Mech. 121 (1982) 27-42.

[16] H.E. Huppert, The propagation of two-dimensional and axisymmetric viscous gravity currents over a rigid horizontal surface, J. Fluid Mech. 121 (1982) 43-58.

[17] V. Di Federico, S. Malavasi, S. Cintoli, Viscous spreading of non-Newtonian gravity currents on a plane, Meccanica 41 (2006) 207-217.

[18] J.Z. Liang, Determination of the entry region length of viscoelastic fluid flow in a channel, Chem. Eng. Sci. 53 (1998) 3185-3187.

[19] N.P. Thien, H.S. Dou, Viscoelastic flow past a cylinder:
Drag coefficient, Comput. Methods Appl. Mech. Eng. 180 (1999) 243-266.

[20] H.E. Huppert, J.E. Simpson, The slumping of gravity currents, J. Fluid Mech. 99 (1980) 785-799.

[21] W. Kozicki, C. Tiu, Non-Newtonian flow through open channels, Can. J. Chem. Eng. 45 (1967) 127-134.

[22] R. Haldenwang, P.T. Slatter, R.P. Chhabra, An experimental study of non-Newtonian fluid flow in rectangular flumes in laminar, transition and turbulent flow regimes, J. South African Inst. Civil Eng. 52 (2010) 11-19.

[23] J. Burger, R. Haldenwang, N. Alderman, Friction factor-reynolds number relationship for laminar flow of non-Newtonian fluids in open channels of different cross-sectional shapes, Chem. Eng. Sci. 65 (2010) 3549-3556.

[24] J.L. Briggs, J.F. Steffe, Mixer viscometer constant (k’) for the Brookfield small sample adapter and flag impeller, J. Texture Studies 27 (1996) 671-677. 